\title{
Polarization VLBI Observations of a Complete Sample of North- ern BL Lacertae Objects
}

A. B. Pushkarev

Sternberg Astronomical Institute, 119899 Moscow, Russia

D. C. Gabuzda

Astro Space Center, Lebedev Physical Institute, 117924 Moscow, Russia

\begin{abstract}
The polarization electric vectors in the VLBI jets of BL Lacertae objects are typically aligned with the jet structure. If the jet radio emission is optically thin synchrotron emission, this implies that the magnetic field is perpendicular to the jet, usually interpreted as a signature of shocks. The distribution of polarization position angles in the VLBI core components appears to be bimodal, with the polarization angles either aligned with or perpendicular to the jet direction. In order to study the origin of this characteristic polarization structure, we have made VLBI polarization observations of all 34 sources in the Kühr and Schmidt sample of BL Lacertae objects.
\end{abstract}

\section{Introduction}

Previous VLBI polarization observations of BL Lacertae objects have shown certain characteristic properties on parsec scales: one-sided core-jet structure with superluminal motions with typical speeds on average lower than those in quasars, jet electric vector $\chi$ parallel to the local jet direction $\theta$, and a tendency for the core $\chi$ values to lie either parallel or perpendicular to the jet axis (Gabuzda et al. 1994 and references therein). The tendency for the jet $\chi$ vectors to align with the jet direction implies that the associated magnetic field is transverse, since there is clear evidence that the jet components are typically optically thin (their degree of polarization can range up to $60 \%$ ): this may indicate the presence of relativistic shocks (in which the transverse magnetic field is amplified by compression (Hughes, Aller, \& Aller 1989; Laing 1980)), which we observe as distinct jet knots. We present here some results for the first epoch of our global VLBI/VLBA polarization study of the sample of northern BL Lacertae objects defined by Kühr \& Schmidt (1990).

\section{Results}

The histograms show the distribution of $|\chi-\theta|$ for the jet and core components in all sources in the Kühr and Schmidt sample for which we have detected polarization. Integrated (VLA core) rotation measures have been applied to the $\chi$ values when they are known. For the jet components, $\chi$ is aligned with $\theta$ in about $75 \%$ of cases, confirming the tendency observed earlier for less systematic studies of BL Lacertae objects (Gabuzda et al. 1994 and refs therein). A few of the objects in the sample have $\chi$ aligned with the jet direction in some components (common for BL Lacertae objects) and transverse to the jet in other components (typical for quasars). The bimodal nature of the distribution of core $\chi$ values suggested previously (Gabuzda et al. 1994) is also clearly visible. 
KUHR \& SCHMIOT BL_AC JETS

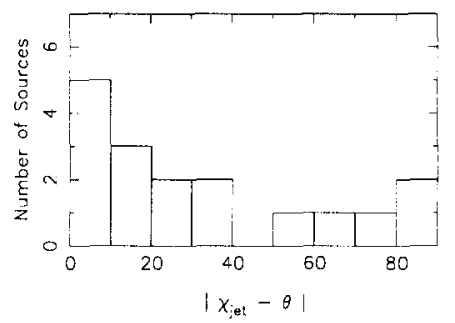

KUHR \& SCHMIOT BL LAC CORES

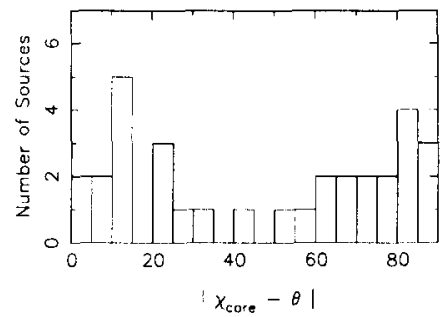

Note that $0745+241$ and $1308+326$, which have $\left|\chi_{\text {core }}-\theta\right|$ values of $85^{\circ}$ and $76^{\circ}$ have some "quasar-like" properties (Murphy, Browne, \& Perley 1993; Hook et al. 1996; Gabuzda et al. 1993), and may be either misclassified quasars or transition objects. In addition, $5 / 17$ sources in the $\left|\chi_{j e t}-\theta\right|$ histogram and $9 / 32$ in the $\left|\chi_{\text {core }}-\theta\right|$ histogram have unknown rotation measures, so that their $\chi$ values are uncertain. We are currently reducing VLA observations at 18 and 20 $\mathrm{cm}$ that will yield VLA core rotation measure values for the Kühr and Schmidt sample sources for which such values have not been previously published; this will resolve most of the possible ambiguity in interpreting the $\chi$ distributions presented here.

Second epoch observations for about a third of the Kühr and Schmidt BL Lacertae objects have already been published (Gabuzda et al. 1994 and refs therein); we are currently analyzing second-epoch $6 \mathrm{~cm}$ global VLBI polarization observation for the remaining sources in parallel with third-epoch data recently obtained on the VLBA at 2, 3.6, and $6 \mathrm{~cm}$. We are also engaged in a project to obtain deep, high resolution $6 \mathrm{~cm}$ VLA images of the sample sources for which these are not yet available (Kollgaard et al. 1992, for example), to try to elucidate the relationship between the parsec-scale and kiloparsec-scale properties of this sample of BL Lacertae objects. A more complete analysis of the results presented here are in preparation for submission to Monthly Notices of the Royal Astronomical Society.

Acknowledgments. We thank the workshop organizers for financial support, and the European VLBI Network and NRAO for their allocation of observing time. The National Radio Astronomy Observatory is a facility of the National Science Foundation, operated under a cooperative agreement by Associated Universities, Inc.

\section{References}

Gabuzda, D. C., et al. 1994. ApJ, 435, 140-161.

Gabuzda, D. C., et al. 1993. ApJ, 410, 39-43.

Hughes, P. A., Aller, H. D., \& Aller, M. F. 1989. ApJ, 341, 54-79.

Hook, I. M., et al. 1996. MNRAS, 282, 1274-1298.

Kollgaard, R. I., et al. 1992. $A J$, 104, 1687-1705.

Kühr, H., \& Schmidt, G. D. 1990. $A J$, 99, 1-6.

Laing, R. A. 1980. MNRAS, 193, 427-437.

Murphy, D. W., Browne, I. W. A., \& Perley, R. A. 1993. MNRAS, 264, 298-318.

Roberts, D. H., Wardle, J. F. C., \& Brown, L. F. 1994. ApJ, 427, 718-744. 